\title{
OBTENÇÃO DE PETIT SUISSE COM BAIXO TEOR DE LACTOSE E ADIÇÃO REDUZIDA DE AÇÚCARES
}

\section{Obtaining Petit Suisse with low amount of lactose and reduced addition of sugars}

\begin{abstract}
Isis Rodrigues Toledo Renhe ${ }^{l^{*},}$, Júlia d'Almeida Francisquini ${ }^{2}$, Danielle Braga Chelini Pereira ${ }^{3}$, Rodrigo Stephani ${ }^{4}$, Antônio Fernandes de Carvalho ${ }^{2}$, Italo Tuler Perrone ${ }^{5}$
\end{abstract}

\section{RESUMO}

Considerando-se as atuais tendências de mercado e orientações do governo brasileiro para a diminuição dos teores de açúcar nos produtos industrializados, faz-se necessária a adaptação das tradicionais tecnologias por outras que permitam modificações nos produtos que atendam à nova realidade sem, contudo, perder em qualidade. $\mathrm{O}$ desenvolvimento do presente trabalho teve como objetivo atender ao crescente público que procura por produtos com baixo teor de lactose, em função do crescimento do número de pessoas intolerantes. Associada a essa diminuição da lactose, esperava-se reduzir a quantidade de sacarose adicionada. Os resultados mostraram que a redução proposta de $20 \%$ na adição de açúcar não foi suplantada pela hidrólise da lactose e os produtos foram sensorialmente diferentes. Os produtos também apresentaram valores estatisticamente diferentes para EST, açúcares redutores, açúcares não redutores e acidez. Apesar das diferenças, os resultados indicam que esse é um produto com potencial e que se pode chegar a um produto mais próximo do original com pequenas alterações na tecnologia de obtenção.

Palavras-chave: queijo; lactase; lactose; redução de açúcar.

1 Empresa de Pesquisa Agropecuária de Minas Gerais, Instituto de Laticínios Cândido Tostes (EPAMIG/ILCT), Rua Tenente Luiz de Freitas, 116, 36045-560, Juiz de Fora, MG, Brasil. E-mail: isis@epamig.br

2 Universidade Federal de Viçosa, Departamento de Tecnologia de Alimentos, Viçosa, MG, Brasil.

3 Laticínios Bela Vista, Bela Vista de Goiás, GO, Brasil.

5 Universidade Federal de Juiz de Fora, Departamento de Química, Juiz de Fora, MG, Brasil.

5 Universidade Federal de Juiz de Fora, Departamento de Ciências Farmacêuticas, Juiz de Fora, MG, Brasil.

* Autor para correspondência. 


\begin{abstract}
Considering the actual marketing tendencies and the Brazilian's governmental guidelines on reduction of sugar levels in industrial products, it is necessary to adapt the traditional technologies for others which allow products modification according to the new reality without, however, losing quality. The development of this work aimed to comply with the consumers who are looking for products with low levels of lactose, as consequence of the increase of intolerant people. Together with the reduction of lactose, it was also expected to reduce the amount of added sugar. The results showed that the proposed reduction of $20 \%$ in added sugar was not supplanted by lactose hydrolysis and the products were considered different. The products also showed statistically different values for total solids, reducing sugars, non reducing sugars and acidity. Despite the differences, the results indicate that this is a product with potential and that it is possible to achieve a product closer to the original with small changes on the obtaining technology.
\end{abstract}

Keywords: Petit Suisse; lactose; lactase; sugar reduction.

\section{INTRODUÇÃO}

A dieta é o principal foco das estratégias de saúde pública na manutenção da saúde ao longo da vida, prevenindo precocemente doenças crônicas como distúrbios gastrointestinais, doenças cardiovasculares, câncer e osteoporose, dentre outros (LOPEZRUBIO et al., 2006). Pessoas que têm uma ingestão adequada de gordura, proteínas, cálcio, magnésio e potássio (todos componentes presentes nos lácteos) são menos propícias a sofrer com hipertensão e fumo (MACDONALD, 2008). Quando associado com grãos, carne, vegetais e frutas, os produtos lácteos são considerados alimentos de alta densidade de nutrientes, ou seja, disponibilizando muitos nutrientes com relativamente baixo conteúdo energético (STEIJNS, 2008).

Considerando a importância nutricional dos produtos lácteos, a otimização e o desenvolvimento de produtos diferenciados são fundamentais para atender as demandas do mercado e aumentar a competitividade das empresas.

O produto Petit Suisse é consumido principalmente pelo público infantil e, por isso, muitas vezes é alvo de críticas pelos seus altos teores de lipídeos e/ou açúcares. Ele é introduzido bem cedo na alimentação das crianças, juntamente com os iogurtes e os leites fermentados (VEIGA et al., 2000; SPINELLI et al., 2001). Para melhorar a imagem desse produto, que algumas vezes é considerado supérfluo por profissionais da saúde, têm sido desenvolvidas alternativas para adultos e crianças que, por motivo de estética ou saúde, precisam ingerir produtos com baixos teores de açúcar, gordura e, muitas vezes, de baixo valor calórico (SOUZA et al., 2010). Outro foco de pesquisas é o enriquecimento do queijo Petit Suisse e de outros lácteos com probióticos e prebióticos (CARDARELLI; SAAD, 2003; MARUYAMA et al., 2006; PEREIRA et al., 2016). Em função dessas pressões, observa-se que nos últimos anos as grandes empresas fabricantes desse queijo têm feito alterações em suas fórmulas, tais como adição de vitaminas e minerais, e substituição de corantes sintéticos por naturais.

No Brasil, a fabricação de queijo Petit Suisse utiliza centrífuga para efetuar o processo de separação da massa, produzindo o 
queijo quark, que é a base utilizada, juntamente com açúcar, creme e frutas, para produzir o Petit Suise (VEIGA; VIOTTO, 2001). A centrífuga é responsável pela realização da tradicional etapa de dessoragem, que contribui para a diminuição do rendimento desse produto e para geração de soro de leite. Uma das formas de aumentar o rendimento do queijo Petit Suise e reduzir a produção de soro de leite é eliminar a etapa de dessoragem do processo de produção, produzindo o queijo Petit Suise com retenção de soro (SOUZA et al., 2012).

Para eliminação desta etapa e obtenção de um produto com retenção de soro faz-se necessária a adição de ingredientes, tais como os hidrocolóides. Esses são polissacarídeos e proteínas amplamente utilizados nas indústrias como agentes de corpo, gelificação, estabilizantes de espumas, inibidores de formação de cristais de gelo e açúcar, entre outras funções (PHILLIPS; WILLIAMS, 2000). Os hidrocolóides podem interagir com as proteínas do leite, gerando um produto final com estabilidade e consistência diferenciadas. A produção do queijo Petit Suisse com retenção do soro pode representar uma grande vantagem dentro dos programas de redução de resíduos das indústrias de laticínios do país e também para o aproveitamento total dos nutrientes contidos no leite (SOUZA et al., 2012).

A hidrólise da lactose à glicose e galactose no leite reduz de 20 a $40 \%$ a necessidade de sacarose para a produção de derivados açucarados, o que propicia uma redução de $10 \%$ do valor energético do produto. A utilização de leite hidrolisado para a produção de leites fermentados e queijos acelera o processo de acidificação, reduzindo o tempo de fabricação e acelerando a desenvolvimento de estrutura e flavor em queijos (TUCKER, 1995). Outro ponto importante na redução dos teores de lactose é atender o público intolerante a este carboidrato. Estima-se que cerca de
$70 \%$ da população mundial apresenta essa síndrome, mas os valores variam regionalmente, podendo ir de 2 a $100 \%$ dependendo da etnia (GONZÁLEZ, 2007).

O objetivo desse trabalho foi desenvolver um produto com menor teor de lactose para atender ao número crescente de pessoas intolerantes e avaliar a composição físicoquímica, perfil de textura e sensorial. Além disso, esperava-se aproveitar o aumento de dulçor causado pela degradação da lactose em glicose e galactose para a diminuição da adição de sacarose no produto. Dessa forma o produto atenderia também a Resolução CNS N 408 (ANVISA, 2008), bem como o documento de referência para guias de boas práticas nutricionais da ANVISA (ANVISA, 2012). Nesse último documento é ressaltado que se deve evitar o consumo excessivo de açúcar e que não deve ser estimulada a substituição deste ingrediente por edulcorantes (adoçantes).

\section{MATERIAL E MÉTODOS}

\section{Formulação dos produtos}

Os produtos foram fabricados em três repetições, com dois tratamentos distintos. O controle foi produzido um Petit Suisse segundo a tecnologia de formulação, com ausência de dessoragem, que tem sido amplamente difundida no mercado. Nesse processo o leite $\left(65,42 \% \mathrm{~m} \cdot \mathrm{m}^{-1}\right)$ foi adicionado de $8,5 \% \mathrm{~m} \cdot \mathrm{m}^{-1}$ de creme ( $36 \%$ de gordura), sacarose $\left(15 \% \mathrm{~m} \cdot \mathrm{m}^{-1}\right)$, agentes de corpo compostos por hidrocolóides (Tecgem AA772, $6 \% \mathrm{~m} \cdot \mathrm{m}^{-1}$ ), polpa (Tecgem AA 21.220 , $5 \% \mathrm{~m} \cdot \mathrm{m}^{-1}$ ) e citrato de sódio $0,08 \% \mathrm{~m} \cdot \mathrm{m}^{-1}$. Estes ingredientes foram misturados e aquecidos até temperatura de $90{ }^{\circ} \mathrm{C}$ por 3 minutos, sendo homogeneizado a pressão de 200 bar quando atingida a temperatura de $50{ }^{\circ} \mathrm{C}$. $\mathrm{O}$ produto formulado foi fermentado com fermento termofílico (MOS 062 E - Sacco) 
composto pelos microrganismos Lactococcus lactis subsp. lactis, Lactococcus lactis subsp. cremoris e Streptococcus thermophilus, a 38 $\pm 2^{\circ} \mathrm{C}$ até $\mathrm{pH}$ próximo de 4,6 . Após atingir o final da fermentação, o produto foi resfriado e texturizado em homogeneizador a pressão de 50 bar, para atingir a textura final desejada no produto.

O segundo tratamento seguiu fabricação semelhante, diferenciando-se pela adição da enzima lactase (SternEzym 21.901L) no início da fermentação, juntamente com o fermento. Foi empregada a dose recomendada pelo fabricante, a concentração de 0,05\% para cada $1 \mathrm{~g}$ de lactose. Foi feita ainda a diminuição de sacarose em $20 \%$ com relação ao controle.

\section{Análises físico-químicas e sensoriais}

A análise centesimal foi realizada, em duplicata, para avaliação dos seguintes atributos: açúcares redutores e não redutores, segundo Wolfschoon-Pombo \& Casagrande (1982); estrato seco total (EST): método gravimétrico (Pereira et al., 2000); atividade de água: empregando Aqualab série 3; acidez (\% de ácido lático) e $\mathrm{pH}$ (medidor de $\mathrm{pH}$ ).

$\mathrm{O}$ perfil de textura foi determinado em equipamento Analisador de Textura CT3 (Brookfield). As condições utilizadas nos testes foram: tipo de teste: Analise do Perfil de Textura (TPA); velocidade de teste: $1,0 \mathrm{~mm} / \mathrm{s}$; distancia de compressão: $10 \mathrm{~mm}$; força mínima de contato: $10,0 \mathrm{~g}$; probe utilizada: cilindro de acrílico de altura: $20 \mathrm{~mm}$; largura: $40 \mathrm{~mm}$; e diâmetro: $38,1 \mathrm{~mm}$. Os dados foram coletados no programa "TexturePro CT V1.2".

Oitenta e quatro consumidores, de ambos os sexos e com idades variando entre 18 e 71 anos, participaram do teste triangular $(\beta=0,05, \alpha=0,10)$ (MEILGAARD et al., 2007), recrutados entre a equipe de colaboradores e estudantes do Instituto de Laticínios Cândido Tostes. O critério de exclusão no teste sensorial foi possuir alergia a proteína do leite de vaca. Os consumidores avaliaram o queijo Petit Suisse após 7 dias da data de produção a uma temperatura de $8{ }^{\circ} \mathrm{C} \pm 3{ }^{\circ} \mathrm{C}$, empregando escala hedônica de 9 pontos (MEILGAARD et al., 2007). A análise de frequência dos escores hedônicos para cada formulação de queijo Petit Suisse foi realizada de acordo com as seguintes faixas de aceitação:

- Rejeição: escores de 1 a 4, indicando que os consumidores desgostaram da amostra

- Indiferentes: escores 5, indicando indiferença dos consumidores

- Aceitação: escores de 6 a 9, indicando que os consumidores gostaram da amostra.

As amostras foram servidas em copos plásticos com capacidade para $30 \mathrm{~mL}$, nos quais aproximadamente $25 \mathrm{~g}$ do produto era fornecida, recebendo codificação randômica com 3 algarismos, apresentados de forma monádica. Os dados foram analisados por meio de análise de variância (ANOVA).

\section{RESULTADOS E DISCUSSÃO}

A Tabela 1 apresenta as médias de composição das amostras dos dois tratamentos. As amostras foram estatisticamente diferentes $(\mathrm{p}<0,05)$ quanto aos quesitos EST, acidez, açúcares não redutores e redutores. Esses resultados mostram-se coerentes com o fato da redução expressiva de sacarose $(20 \%)$ na formulação do tratamento, que altera os sólidos totais e os açúcares não redutores. A adição de lactose altera os valores dos açúcares redutores, pois, de uma molécula redutora, lactose, passa-se a duas moléculas de açúcar redutor, glicose e galactose. Entretanto, ressalta-se que o teor de açúcares totais não apresentou diferença significativa $(\mathrm{p}>0,05)$ exatamente pelo aumento de 
moléculas de açúcar resultante da conversão da lactose.

Os resultados de proteína e gordura não são apresentados, pois seus valores apresentam-se incoerentes com a formulação realizada, considerando-se o balanço de massa das quantidades de matéria prima empregada e suas composições centesimais. Em alguns casos a amostra foi submetida a mais de 15 repetições da mesma análise, trocando-se o analista e os reagentes para eliminar possíveis erros de execução e problemas com reagentes. Tais resultados levantam uma demanda por adequações nas metodologias analíticas atuais existentes. Sabe-se que o uso de gomas atinge seus objetivos tecnológicos exatamente pela interação com constituintes do alimento e acreditamos que essas interações têm afetado a separação e o fracionamento para a quantificação das moléculas em análise. O trabalho de Paixão e colaboradores (2011) também apresenta valores abaixo dos esperados e exigidos pela legislação para proteína e gordura. Essa afirmação se baseia em resultados de análises anteriores de produtos de mercado, onde foram encontrados resultados coerentes nos produtos onde é sabido que é empregada a tecnologia tradicional de centrifugação para a dessoragem. Produtos onde há indícios do uso de formulações para evitar a etapa de separação do soro apresentaram resultados incoerentes com o esperado e até mesmo com a legislação. Levanta-se a hipótese de nesses produtos estarem ocorrendo interações entre os constituintes e os ingredientes até o momento pouco exploradas e que afetam o preparo tradicional das amostras para análise.

Apesar de não apresentar diferença estatística significativa $(\mathrm{p}>0,05)$ em todos os resultados das análises de perfil de textura, alguns provadores destacaram a percepção de diferença nesse atributo entre as amostras. Para vários julgadores houve diferença entre a viscosidade dos produtos. Essa diferença pode ser ajustada pela alteração dos hidrocolóides adicionados, bem como suas proporções (MARUYAMA et al., 2006).

As amostras apresentaram diferença sensorial estatisticamente significativa pelo teste triangular $(\beta=0,05, \alpha=0,10)$. Observouse que a hidrólise de lactose em glicose e galactose, que deveria aumentar o dulçor do produto, não foi suficiente para suplantar a

Tabela 1 - Médias observadas da composição centesimal das formulações de queijos petit suisse

\begin{tabular}{lccc}
\hline & \multicolumn{3}{c}{ Formulações } \\
\hline Variável & Controle & Tratamento & $p$-valor \\
\hline Aw & 0,973 & 0,971 & 0,01 \\
EST $\left(\% \mathrm{~m} \cdot \mathrm{m}^{-1}\right)$ & 4,64 & 4,32 & 0,01 \\
Acidez $\left({ }^{\circ} \mathrm{D}\right)$ & 35,3 & 31,9 & 0,02 \\
Açúcares redutores $\left(\% \mathrm{~m} \cdot \mathrm{m}^{-1}\right)$ & 26 & 29,8 & 0,01 \\
Açúcares não redutores $\left(\% \mathrm{~m} \cdot \mathrm{m}^{-1}\right)$ & 5,4 & 11,2 & 0,02 \\
Açúcares totais $\left(\% \mathrm{~m} \cdot \mathrm{m}^{-1}\right)$ & 16,9 & 10,5 & 0,02 \\
Dureza & 21,6 & 21,8 & 0,24 \\
Adesividade & 599,75 & 441,5 & 0,10 \\
Elasticidade & 15,2 & 14,6 & 0,23 \\
\hline
\end{tabular}


redução de $20 \%$ na quantidade de sacarose adicionada na formulação do produto, segundo os dados descritos em literatura (TUCKER; WOODS, 1995). Como complementação da avaliação sensorial, foi feita a análise de preferência por meio de escala hedônica para verificar a aceitação dos produtos. A análise estatística mostrou que a diferença entre as tecnologias afetou a aceitação dos produtos, com média 7,6 para o controle e 6,5 para o tratamento. As médias foram estatisticamente diferentes em nível de $5 \%$ de probabilidade. De acordo com as observações colocadas pelos julgadores, percebeu-se que o açúcar é importante para encobrir a acidez característica do produto, pois dulçor e acidez estiveram entre os quesitos mais citados como fator de diferenciação entre as amostras.

A Figura 1 apresenta a frequência dos escores na análise sensorial de aceitação por escala hedônica. Observa-se que apesar da diferença estatística entre as médias, o produto com redução de sacarose continua apresentando elevado percentual de aceitação.

\section{CONCLUSÕES}

A formulação empregada no expe- rimento possibilitou a obtenção de queijo Petit Suisse com teor reduzido em lactose e com composição próxima ao produto tradicional. Apesar da menor aceitação, o produto adicionado de lactase e com menor teor de açúcar ainda pode ser considerado viável pela sua avaliação de aceitação entre gostei ligeiramente e gostei moderadamente. Estudos adicionais são necessários para verificar se a interrupção da fermentação em $\mathrm{pH}$ mais elevado reduziria o impacto da diminuição do açúcar. Outro fator que poderia ser avaliado é o uso do parâmetro acidez no lugar do $\mathrm{pH}$ para determinar o final da fermentação do produto. A importância de avaliações adicionais está na consonância do desenvolvimento desse produto com as diretrizes atuais de diminuição do consumo de açúcares, gorduras e sódio, entre outros, do Governo Federal, através da Agência Nacional de Vigilância Sanitária.

\section{AGRADECIMENTOS}

À FAPEMIG pelo financiamento do projeto e bolsas concedidas aos autores. Às empresas Gemacom Tech, Sacco do Brasil e Stern Ingredientes pelo fornecimento das amostras para a elaboração dos produtos.

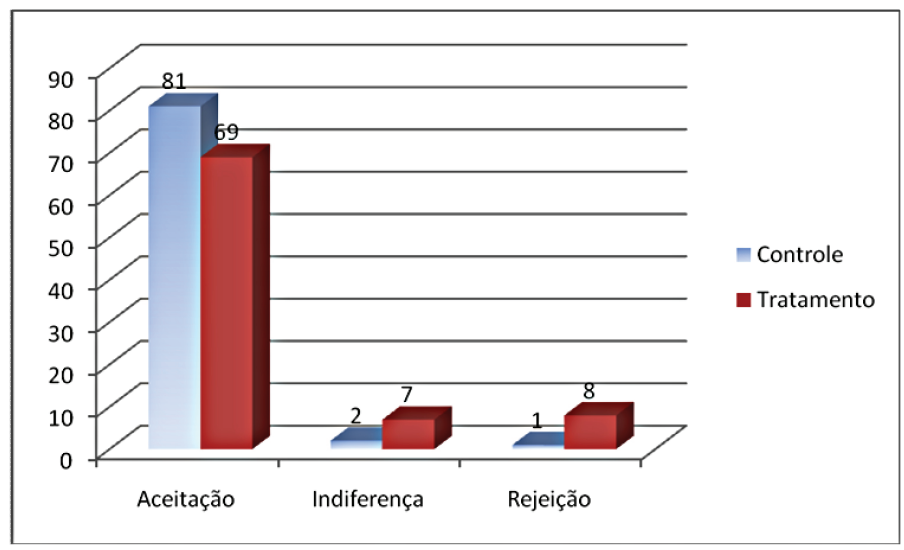

Figura 1 - Frequência dos escores na análise sensorial de aceitação por escala hedônica 


\section{REFERÊNCIAS}

AGÊNCIA NACIONAL DE VIGILÂNCIA SANITÁRIA (ANVISA). Resolução CNS $\mathrm{N}^{\circ} 408$ de 11 de dezembro de 2008. Aprova diretrizes para a promoção da alimentação saudável com impacto na reversão da epidemia de obesidade e prevenção das doenças crônicas não transmissíveis. Diário Oficial da República Federativa do Brasil, Brasília, 09 de março de 2009.

AGÊNCIA NACIONAL DE VIGILÂNCIA SANITÁRIA (ANVISA). Guia de boas práticas nutricionais. Documento de Referência. Brasília: Agência Nacional de Vigilância Sanitária, 2012. 12 p.

CARDARELLI, H. R.; SAAD, S. M. I. . Avaliação microbiológica de diferentes formulações de queijo petit-suisse probiótico durante o armazenamento refrigerado. In: SIMPÓSIO LATINO AMERICANO DE CIÊNCIA DE ALIMENTOS, 5., 2003, Campinas. Anais... Campinas: UNICAMP, 2003. 1 CD-ROM.

PHILLIPS, G. O.; WILLIAMS, P. A. Handbook of hydrocolloids. Boca Raton: CRC Press, 2000. 450p.

GONZÁLES, F. A. Intolerancia a la lactosa y otros disacáridos. Acta gastroenterologica latinoamericana, v. 18, n. 2, p. 152-156, 2007.

LOPEZ-RUBIO, A.; GAVARA，R.; LAGARON, J. M. Bioactive packaging: turning foods into healthier foods through biomaterials. Trends in Food Science \& Technology, v. 17, p. 567-575, 2006.

MACDONALD, H. B. Dairy nutrition: What we knew then what we know now. International Dairy Journal, v. 18, p. 774$777,2008$.
MARUYAMA, L. Y. et al. Textura instrumental de queijo Petit-Suisse potencialmente probiótico: influência de diferentes combinações de gomas. Ciência e Tecnologia de Alimentos, v. 26, n. 2, p. 386-393, 2006.

MEILGAARD, M.; CIVILLE, G. V.; CARR, $B$. T. Sensory evaluation techniques. Boca Raton: CRC Press, 2007. 448p.

PAIXÃO, M. G. et al. Caracterização físico-química de queijos Petit Suisse comercializados na região de Lavras-MG e adequação dos rótulo quanto à legislação. Revista do Instituto de Laticínios Cândido Tostes, v. 66, n. 383 , p. $05-12,2011$.

PEREIRA, D. B. C. et al. Físico-química do leite e derivados - métodos analíticos. $2^{\mathrm{a}} \mathrm{ed}$. Juiz de Fora, 2001. 234 p.

PEREIRA, E. P. R. et al. Effect of incorporation of antioxidants on the chemical, rheological, and sensory properties of probiotic petit suisse cheese. Journal of Dairy Science, v. 99 , n. 3, p. 1762-1772, 2016.

SPINELLI, M. G. N.; SOUZA, S. B.; SOUZA, J. M. P. Consumo, por crianças menores de um ano de idade, de alimentos industrializados considerados supérfluos. Pediatria Moderna, v. 37, n. 4, p. 22-27, 2001.

SOUZA, V. R. et al. Elaboração de queijo Petit Suisse sabor morango de baixo valor calórico. Revista do Instituto de Laticínios Cândido Tostes, v. 65, n. 374, p. 49-58, 2010.

SOUZA, V. R. et al. Efeito da concentração de gordura nas propriedades físicas, químicas e sensoriais do queijo Petit Suisse elaborado com retenção de soro. Revista do Instituto de Laticínios Cândido Tostes, v. 67, n. 386, p. 20-28, 2012 
STEIJNS, J. M. Dairy products and health: Focus on their constituents or on the matrix? International Dairy Journal, v. 18, p. 425435, 2008.

TUCKER, G. A.; WOODS, L. F. J. Enzymes in food processing. $2^{\mathrm{a}}$ ed. New York: Blackie Academic \& Professional, 1995. 319 p.

VEIGA, P. G. et al. Caracterização química, reológica e aceitação sensorial do queijo petit suisse brasileiro. Ciência e Tecnologia de Alimentos, v. 20, n. 3, p. 349-357, 2000.
VEIGA, P. G.; VIOTTO, W. H. Fabricação de queijo Petit Suisse por ultrafiltração de leite coagulado. Efeito do tratamento térmico do leite no desempenho da membrana. Ciência e Tecnologia de Alimentos, v. 21, n. 3, p. 267-272, 2001.

W O L F S C H O ON - P O M B O A. F. ; CASAGRANDE, H. de R. Nova técnica para a determinação da lactose e sacarose no doce de leite. Revista do Instituto de Laticínios Cândido Tostes, n. 222, v. 37, p. 03-07, 1982. 